Check for updates

Cite this: RSC Adv., 2019, 9, 17801

Received 6th March 2019 Accepted 27th May 2019 DOI: 10.1039/c9ra01717h rsc.li/rsc-advances

\section{Local delivery of FTY720 and NSCs on electrospun PLGA scaffolds improves functional recovery after spinal cord injury}

\author{
Weijian Kong, (D) $\dagger^{\star a}$ Zhiping Qi, (D) $\dagger^{\star a}$ Peng Xia, ${ }^{a}$ Yuxin Chang, ${ }^{a}$ Hongru Li, ${ }^{a}$ \\ Yunpeng Qu, ${ }^{\text {b }}$ Su Pan ${ }^{\star a}$ and Xiaoyu Yang ${ }^{\star a}$
}

Spinal cord injury ( $\mathrm{SCl}$ ) is a common issue in the clinic that causes severe motor and sensory dysfunction below the lesion level. FTY720, also known as fingolimod, has recently been reported to exert a positive effect on the recovery from a spinal cord injury. Through local delivery to the lesion site, FTY720 effectively integrates with biomaterials, and the systemic adverse effects are alleviated. However, the effects of the proper mass ratio of FTY720 in biomaterials on neural stem cell (NSC) proliferation and differentiation, as well as functional recovery after $\mathrm{SCl}$, have not been thoroughly investigated. In our study, we fabricated electrospun poly (lactide-co-glycolide) (PLGA)/FTY720 scaffolds at different mass ratios $(0.1 \%, 1 \%$, and $10 \%)$ and characterized these scaffolds. The effects of electrospun PLGA/FTY720 scaffolds on NSC proliferation and differentiation were measured. Then, a rat model of spinal transection was established to investigate the effects of PLGA/FTY720 scaffolds loaded with NSCs. Notably, $1 \%$ PLGA/FTY720 scaffolds exerted the best effects on the proliferation and differentiation of NSCs and $10 \%$ PLGA/FTY720 was cytotoxic to NSCs. Based on the Basso, Beattie, and Bresnahan (BBB) score, HE staining and immunofluorescence staining, the PLGA/FTY720 scaffold loaded with NSCs effectively promoted the recovery of spinal cord function. Thus, FTY720 properly integrated with electrospun PLGA scaffolds, and electrospun PLGA/FTY720 scaffolds loaded with NSCs may have potential applications for $\mathrm{SCl}$ as a nerve implant.

\section{Introduction}

Spinal cord injury (SCI) is a common issue in the clinic that causes severe motor and sensory dysfunction below the lesion level. ${ }^{\mathbf{1 , 2}}$ Moreover, SCI has not been treated effectively in recent years. Effective, impactful solutions for this problem are unavailable. The regeneration of neurons and axons in the lesion area is the key to rehabilitating nerve function. ${ }^{3,4}$ Neural stem cells (NSCs) present in the nervous system have the ability to proliferate and differentiate into functional neurons. ${ }^{5,6}$ After SCI, NSCs are activated immediately and migrate to the lesion site. ${ }^{4}$ However, the hostile pathological microenvironment stimulates NSCs to differentiate into astrocytes and microglial cells rather than mature neurons, potentially causing the formation of a glial scar that hampers axon regeneration. ${ }^{7}$ In addition, the inflammatory and autoimmune responses of the damaged area have an adverse effect on the survival of NSCs and neurons. ${ }^{\mathbf{8 9}}$ Trauma-induced autoimmunity generates

\footnotetext{
${ }^{a}$ Department of Orthopedic Surgery, The Second Hospital of Jilin University, Ziqiang Street No. 218, Changchun, Jilin 130041, PR China. E-mail: yangxiaoy@jlu.edu.cn ${ }^{b}$ Department of Cardiovascular Medicine, The Second Hospital of Jilin University, Ziqiang Street No. 218, Changchun, Jilin 130041, PR China

$\dagger$ Weijian Kong and Zhiping Qi contributed equally to this work.
}

lymphocytes and $\mathrm{T}$ cells around the lesion, resulting in the release of neural self-antigens and subsequent immune reactions. ${ }^{\mathbf{6}, \mathbf{9} 10}$ The use of drugs to suppress inflammatory and immune reactions is a new and effective method.

Fingolimod, also known as FTY720, is one of the most recently developed immunomodulatory medicines that has been approved for the treatment of multiple sclerosis. ${ }^{\mathbf{1 1}}$ FTY720 is a chemical derivative of the fungal metabolite myriocin. Phosphorylated FTY720 antagonizes the sphingosine-1-phosphate (S1P) receptor, which regulates many types of immune-associated cellular processes, such as lymphocyte proliferation and mast cell activation. ${ }^{7,12} \mathrm{~S} 1 \mathrm{P}$ has another mechanism, as it reduces the effectiveness of lymphocytes migration from the thymus to inflammatory sites. ${ }^{\mathbf{1 3 , 1 4}}$ FTY720 reportedly promotes cell survival by inhibiting apoptosis and preventing neuronal death in subjects with neurodegenerative diseases, confirming that FTY720 exerts a clear neuroprotective effect on the central nervous system. ${ }^{12,15}$ However, according to recent studies, the systemic administration of FTY720 might cause adverse effects, including systemic lymphopenia and reproductive and developmental toxicity, such as weight loss. ${ }^{16,17}$ Local delivery might allow FTY720 to directly cross the blood-brain 
barrier, with a higher local concentration but lower plasma concentration.

Currently, different types of biodegradable materials, such as chitosan, poly (lactide-co-glycolide) (PLGA), and collagen, are widely utilized in many fields. ${ }^{18-20}$ PLGA has a great application potential because of its excellent biodegradability, mechanical properties, low immunogenicity and toxicity, and adjustable degradation rates. ${ }^{21}$ At present, PLGA-based nanofibres have made significant contributions to nerve regeneration due to their porous interconnected structures. ${ }^{19,22}$ In addition, PLGA nanofibres have a completely open surface, which is conducive to the transport of water and nutrients needed for cell growth. ${ }^{23,24}$ Although PLGA has many advantages, the hydrophobic surface of PLGA does not accelerate cell adhesion. ${ }^{25}$ The structure of electrospun fibres simulate the scale and high surface area/volume ratio of the extracellular matrix. ${ }^{26}$ Electrospun materials have been shown to facilitate the extension of neuronal process and the recovery of nerve function. ${ }^{26,27}$ Additionally, drugs and materials can be premixed during the preparation of the material, which is conducive to drug delivery ${ }^{28}$ PLGA is also easy to obtain, which is conducive to large-scale applications. Therefore, we chose to establish modified electrospun PLGA microfibre scaffolds as drug transport materials to reduce adverse effects.

Existing studies have explored the effect of FTY720 on NSCs in vitro, ${ }^{29}$ and other studies have explored the therapeutic effect of the combination of FTY720 with biomaterials on related diseases. ${ }^{30-32}$ However, the effects of PLGA/FTY720 scaffolds with different mass ratios on the proliferation and differentiation of NSCs has not been explored. In our study, we cultured NSCs on PLGA/FTY720 scaffolds with different mass ratios to explore the effects of different drug doses on the proliferation and differentiation of NSCs. Moreover, few studies have used NSCs together with PLGA/FTY720 scaffolds. In our study, we loaded NSCs on PLGA/FTY720 scaffolds and investigated the effects of PLGA/FTY720 scaffolds combined with NSCs on the recovery of SCI by constructing a total resection model in rats. We created PLGA/FTY720 electrospun microfibres with different mass ratios and tested their biological properties. The proper mass ratio of PLGA/FTY720 scaffolds for NSC growth and the effects of different materials were examined in vitro. Then, we established a T9 spinal cord transection rat model and implanted different scaffolds in the injury site. Motor sensory functions and histology experiments were used to evaluate the therapeutic effects. Based on the results, electrospun PLGA/ FTY720 fibres with NSCs improve the recovery of SCI both in vivo and in vitro and promote neurogenesis at the lesion site to enhance motor sensory functional rehabilitation.

\section{Materials and methods}

\subsection{Fabrication of PLGA/FTY720 scaffolds}

$\operatorname{PLGA}\left(M_{\mathrm{w}}=40000 \mathrm{~g} \mathrm{~mol}^{-1}\right)$ with a lactic acid : glycolic acid ratio of $80: 20$ was purchased from Changchun Sino Biomaterials Co., Ltd. The method used to manufacture electrospun PLGA nanofibres was described in a previous study. ${ }^{33}$ PLGA $(100 \mathrm{mg})$ and FTY720 (0.1 mg, Gilenya, Novartis) were dissolved in $5 \mathrm{~mL}$ of $1,1,1,3,3,3$-hexafluoroisopropanol (HFIP) to achieve a mass ratio of $0.1 \%$. Then, the mixture was placed in $10 \mathrm{~mL}$ plastic syringes equipped with $0.4 \mathrm{~mm}$ needles. The nanofibres were manufactured at an applied voltage of $20 \mathrm{kV}$ with a voltageregulated DC power supply (DW-P203-1ACFD, Tianjin Dongwen High Voltage Power Supply Plant, China) at a feeding rate of $1.0 \mathrm{~mL} \mathrm{~h}^{-1}$. The distance between the collector and the needle was $10 \mathrm{~cm}$. Electrospun PLGA fibres were collected from the receiving screen. Fibres with different mass ratios of FTY720 and PLGA (1\% and 10\%) were manufactured using the same procedure. Pure PLGA nanofibres were prepared without adding FTY720 to the solution.

\subsection{Characterization}

A scanning electron microscope (SEM, XL30 ESEM-FEG, FEI) was selected to obtain the surface morphology data from the electrospun fibre scaffolds. The static water contact angle was measured to assess the surface wettability and hydrophobicity using the sessile drop method on a contact angle system (VCA 2000, AST).

\section{$2.3 \quad$ NSC cultures}

All animal procedures were performed in accordance with the Guidelines for Care and Use of Laboratory Animals of Jilin University and authorized by the Institutional Animal Care and Use Committee of Jilin University. NSCs were isolated from the cerebral cortex of embryonic day 14.5 (E14.5) SD rats as previously described. ${ }^{25}$ These cells were purified and cultured in T25 culture flasks (Corning, NY, USA) at a density of 50000 cells per $\mathrm{cm}^{2}$ in a humidified atmosphere composed of $5 \% \mathrm{CO}_{2}$ at $37^{\circ} \mathrm{C}$. The growth medium contained NB (Gibco, USA), 2\% B27 neural supplement (Gibco, USA), $20 \mathrm{ng} \mathrm{mL}^{-1}$ epidermal growth factor

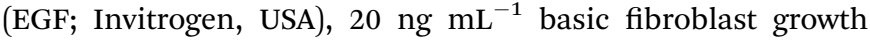
factor (BFGF, Sigma) and $100 \mathrm{ng} \mathrm{mL}^{-1}$ penicillin-streptomycin (Gibco-Invitrogen). Primary NSCs were subcultured every four days, and the medium was replaced every two days. Cells between passages two to five were used in the experiments.

\subsection{Cell proliferation}

Cells were divided into four groups and cultured on PLGA/ FTY720 fibres with different mass ratios at a density of 30000 cells per $\mathrm{cm}^{3}$ to evaluate the effect of FTY720 on NSCs. Pure PLGA nanofibres were included as the control group. Cell proliferation was analysed to determine the effect and proper mass ratio of PLGA/FTY720 electrospun fibre scaffolds. An MTT assay was applied to assess proliferation at 1, 3, and 7 days according to the manufacturer's instructions. Briefly, $50 \mu \mathrm{L}$ of the MTT (Sigma) stock solution in PBS were added to each well, and the cells were incubated at $37^{\circ} \mathrm{C}$ for $4 \mathrm{~h}$. Then, the medium was removed, and $750 \mu \mathrm{L}$ of acidified isopropanol were added to each well. The optical density (OD) was measured at a wavelength of $540 \mathrm{~nm}$ using a Full Wavelength Microplate Reader (Infinite M200, TECAN). NSCs cultured on FTY720 nanofibres with different mass ratios were analyses using this assay. 


\subsection{Immunofluorescence staining}

Immunofluorescence staining was performed to evaluate NSC differentiation on PLGA/FTY720 electrospun fibres with different mass ratios. All four groups of NSCs were cultured for 7 days. Then, the cells were washed with 0.01 M PBS, fixed with $4 \%$ paraformaldehyde for $60 \mathrm{~min}$, extracted with $0.1 \%$ Triton X-100 (Sigma) for $10 \mathrm{~min}$ and blocked with $10 \%$ goat serum (Sigma) for $2 \mathrm{~h}$. An anti-Tuj-1 antibody (1 : 100; Millipore) was added to label neurons, an anti-Oligo antibody (1 : 200; Abcam) was added to label oligodendrocytes and an anti-GFAP antibody (1:150, Millipore) was added to label astrocytes. After an overnight incubation at $4{ }^{\circ} \mathrm{C}$, secondary antibodies were added and incubated for another $2 \mathrm{~h}$ at room temperature. Finally, these cells were stained with DAPI. All stained cells were observed under a confocal laser scanning microscope (FV1000, Olympus).

\subsection{Quantitative real-time PCR analysis}

NSCs cultured on different PLGA/FTY720 fibres for 7 days were also collected to evaluate gene expression. Total RNA was extracted using TRIzol reagent (Invitrogen) according to the manufacturer's protocol. The total RNA concentration and purity were detected using a Nanodrop spectrophotometer (Tecan M200). RNA samples were reverse transcribed into cDNAs for qPCR using a PrimeScript ${ }^{\mathrm{TM}}$ reagent kit with gDNA Eraser (Takara). The primer sequences specific for the target genes used for qRT-PCR are listed in Table 1. The qPCR procedure was started with an initial denaturation at $95{ }^{\circ} \mathrm{C}$ for $10 \mathrm{~min}$, followed by 40 cycles at $95{ }^{\circ} \mathrm{C}$ for $30 \mathrm{~s}, 58{ }^{\circ} \mathrm{C}$ for $1 \mathrm{~min}$, and $72{ }^{\circ} \mathrm{C}$ for $1 \mathrm{~min}$. The expression of each gene was normalized to the expression of the housekeeping gene glyceraldehyde-3-phosphate dehydrogenase (GAPDH).

\subsection{Spinal cord injury model}

All animal procedures were performed in accordance with the Guidelines for Care and Use of Laboratory Animals of Jilin University and authorized by the Institutional Animal Care and Use Committee of Jilin University. Adult female SpragueDawley rats (200-250 g) were purchased from the animal centre of Jilin University and randomly divided into the PLGA group ( $n=8)$, PLGA/FTY720 group $(n=8)$, PLGA-FTY720/NSC group $(n=8)$ and control group $(n=8)$. All animals were endotracheally intubated, and inhalational anaesthesia (2.5\% isoflurane) was maintained during all surgical procedures. A laminectomy was performed at the T9 level using tissue forceps and a rongeur; then, the dura was opened with eye scissors to expose the spinal cord tissue. Venus scissors

Table 1 List of genes and primer nucleotide sequences

\begin{tabular}{lll}
\hline Gene & Forward primer & Reverse primer \\
\hline Tuj-1 & GATCGGAGCCAAGTTCTG & GTCCATCGTCCCAGGTTC \\
GFAP & GCAGACCTTCTCCAACCTG & ACTCCTTAATGACCTCTCCATC \\
Oligo & CGACGCCAAAGAGGAACAG & GCCAAGTTCAGGTCCTGCAT \\
GAPDH & TCGCCAGCCGAGCCA & CCTTGACGGTGCCATGGAAT
\end{tabular}

were chosen to create a $1 \mathrm{~mm}$ gap at the T9 vertebral level. All spinal cord segments, including the spinal cord roots, were completely removed from the gap. After a saline flush and haemostasis, different scaffolds were implanted in the SCI site. PLGA/FTY720 scaffolds were prepared as previously described. ${ }^{25}$ All fibres were rolled into a $3 \mathrm{~mm}$ diameter cylinder. The cylinder was cut transversely into $1 \mathrm{~mm}$-long cylinders, exposed to ultraviolet light for 24 hours and washed with PBS three times prior to use in further experiments. Scaffolds in the PLGA-FTY720/NSC group were immersed in $10 \%$ poly-lysine for $2 \mathrm{~h}$ and washed with PBS three times before NSCs were allowed to adhere. Then, the scaffolds were immersed in culture medium containing 500000 cells per $\mathrm{cm}^{3}$ for 3 days before use. In the control group, the spinal cord was removed, but no implantation was performed. Finally, the muscle and anadesma were sutured and the skin was closed.

\subsection{Assessment of locomotor recovery}

In our study, the Basso, Beattie, and Bresnahan (BBB) locomotor rating scale was adopted to evaluate the functional recovery of hind limbs. All rats were scored weekly during a 4 min period by two individual examiners who were blinded to the animals' treatments.

\subsection{Histological and immunofluorescence staining}

Six weeks after injury, all rats were sacrificed with a lethal dose of pentobarbital and perfused with $200 \mathrm{~mL}$ of $\mathrm{PBS}(\mathrm{pH}=7.4)$ via the aorta, followed by $200 \mathrm{~mL}$ of $4 \%$ paraformaldehyde. The spinal cords were excised from animals and immersed in $4 \%$ paraformaldehyde overnight. The spinal cord tissue was then dehydrated in $30 \%$ sucrose for $48 \mathrm{~h}$. Next, the segments were cut into $15 \mathrm{~mm}$ thick sections and embedded in OCT. The tissue was then subjected to haematoxylin and eosin staining. An antiNeuN antibody (1:200, Abcam) was chosen as the marker for mature neurons. The immunofluorescence staining procedure was described in a previous study. The NIH ImageJ program was used to quantify the area of the cavity in the spinal cord on histology slides and the mean number of NeuN-positive cells per visual field. Cavities located within $3 \mathrm{~mm}$ of the injury centre were examined and measured in a blinded manner. For the analysis of NeuN-positive cell counts, 6 fields from one sample were randomly selected to obtain the average number of NeuN-positive cells.

\subsection{Statistical analysis}

All quantitative data were analysed with GraphPad Prism 5.0 software (GraphPad Software, USA) and presented as means \pm standard deviations. Significant differences were determined using one-way analysis of variance (ANOVA) for more than two variables and $t$ tests for two variables. A value of $P<0.05$ was considered significant. 


\section{Results}

\subsection{Characterization of PLGA nanofibre scaffolds}

The microstructure and average fibre diameter of the PLGA and PLGA/FTY720 fibres with different mass ratios were determined using SEM. As shown in Fig. 1, both PLGA microfibres and PLGA/FTY720 microfibres with different mass ratios were fabricated in random orientations. The pure PLGA nanofibres had the largest mean fibre diameter of $1513 \mathrm{~nm}$. All fibres mixed with FTY720 had smaller diameters than the pure PLGA fibres. In addition, a higher FTY720 mass ratio in microfibres resulted in a smaller diameter. A microfibre with a $10 \%$ mass ratio FTY720 had the smallest diameter of $1040 \mathrm{~nm}$. The change in solution viscosity and ductility probably led to different fibre diameters.

The contact angles of PLGA and different PLGA/FTY720 microfibres were calculated to assess the hydrophilicity of the fibre surface. As shown in Fig. 2, the contact angles of the fibres with FTY720 were much smaller than the pure PLGA microfibres. The mean contact angle of pure PLGA was $114.7^{\circ}$. The

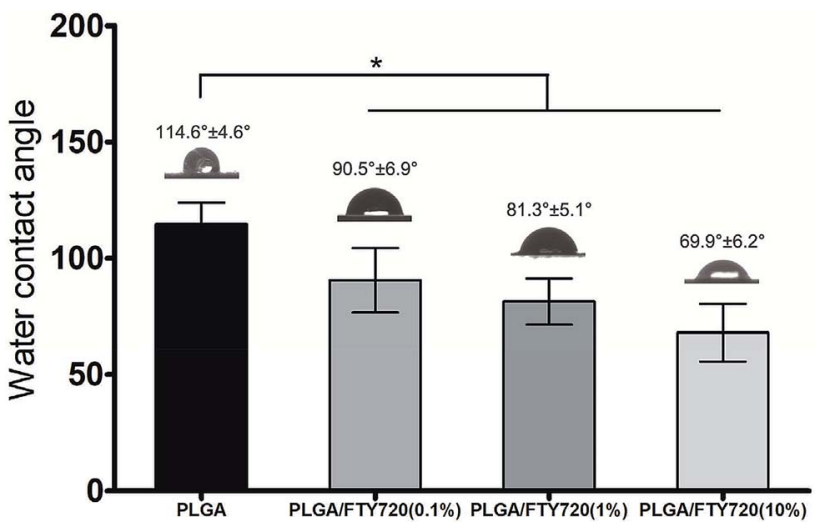

Fig. 2 Water contact angles of PLGA, PLGA/FTY720 (0.1\%), PLGA/ FTY720 (1\%) and PLGA/FTY720 (10\%). (*P<0.05, $n=3)$.

mean contact angle of PLGA/FTY720 scaffolds ranged from $90.4^{\circ}$ to $67.9^{\circ}$. Based on this finding, FTY720 improved the hydrophilicity of the fibre surface when mixed with PLGA microfibres.
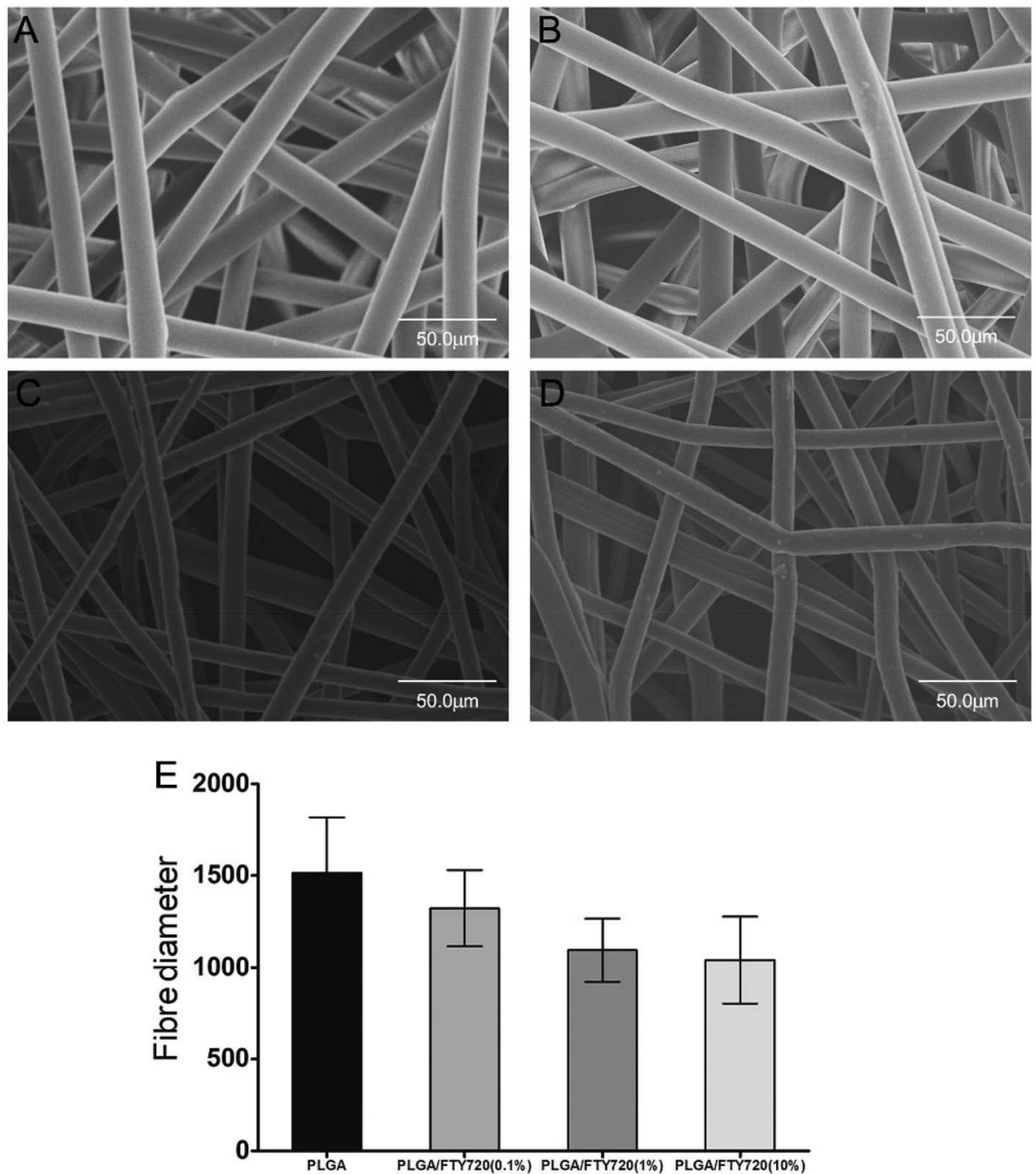

Fig. 1 Scanning electron microscopy images of PLGA (A), PLGA/FTY720 (0.1\%) (B), PLGA/FTY720 (1\%) (C) and PLGA/FTY720 (10\%) (D). The average fibre diameter (E). ( $* P<0.05, n=3)$. 


\subsection{Proliferation of NSCs}

The proliferation of NSCs on different microfibres was measured using the MTT method at 1, 3, and 7 days. As shown in Fig. 3, significant differences in the proliferation of NSCs were not observed among these four groups after 1 day. The OD values of the PLGA/FTY720 (0.1\%) group and PLGA/FTY720 (1\%) group were significantly higher than the PLGA group and PLGA/FTY720 (10\%) group, respectively, at 3 days. Furthermore, after NSCs were cultured for 7 days, the OD value of the PLGA/ FTY720 (1\%) group was significantly higher than the PLGA group. In addition, the OD value of the PLGA/FTY720 (10\%) group was lower than the PLGA group at 7 days.

\subsection{NSC differentiation}

The ability of different fibres to alter NSC differentiation was assessed using qRT-PCR and immunofluorescence staining. GFAP, Tuj-1 and Oligo antibodies were chosen to evaluate cell differentiation in our study. The immunofluorescence staining presented in Fig. 4 shows that the PLGA/FTY720 (10\%) group had the lowest expression of the GFAP protein among the groups. Increased expression of the Oligo protein was observed in the PLGA/FTY720 (1\%) and PLGA/FTY720 (10\%) groups compared with the PLGA and PLGA/FTY720 (0.1\%) groups. In addition, the PLGA/FTY720 (1\%) group and PLGA/FTY720 (10\%) group expressed Tuj-1 at higher levels than the PLGA and PLGA/ FTY720 (0.1\%) groups. As shown in Fig. 5, the expression of the GFAP mRNA gradually decreased with increasing drug concentrations, and the expression levels in the PLGA/FTY720 (1\%) and PLGA/FTY720 (10\%) groups were significantly lower than the levels in the PLGA and PLGA/FTY720 (0.1\%) groups. A significant difference in the expression of the Tuj-1 mRNA was not observed between the groups, but a slight increase was observed as the FTY720 mass ratio increased. The highest expression of the Oligo mRNA was observed in the PLGA/ FTY720 (1\%) group, and no significant differences were

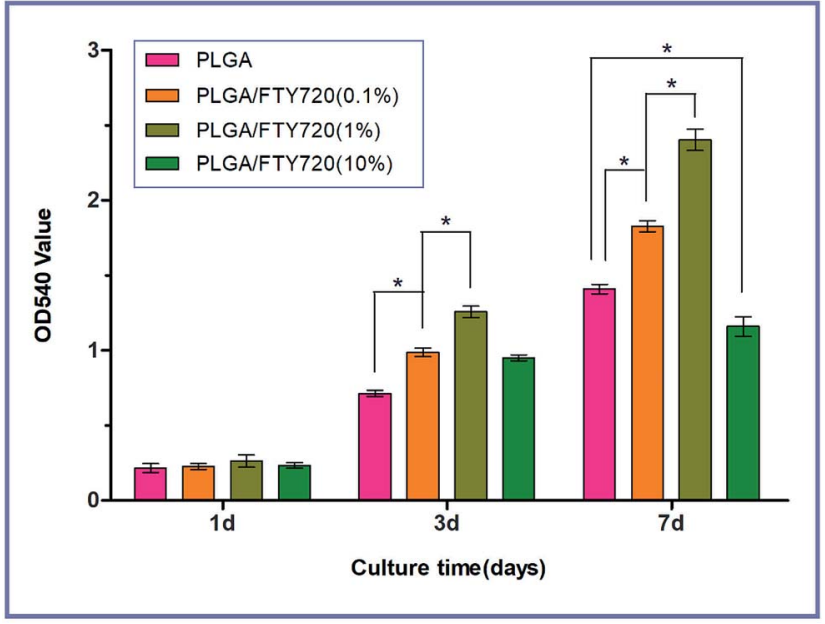

Fig. 3 Proliferation of NSCs cultured with PLGA, PLGA/FTY720 (0.1\%), PLGA/FTY720 (1\%) and PLGA/FTY720 (10\%) for 1, 3, and 7 days in vitro. $(* P<0.05, n=3)$. observed between the PLGA, PLGA/FTY720 (0.1\%) and PLGA/ FTY720 (10\%) groups $(P>0.05)$.

\subsection{Assessment of locomotor recovery}

We established a T9 transection in a rat model to evaluate the therapeutic effects of the scaffolds on SCI in vivo. The BBB score was analysed for up to 6 weeks after injury. As shown in Fig. 6 , the BBB score of the PLGA-FTY720/NSC group revealed significantly increased functional recovery compared with the PLGA group and PLGA-FTY720 group. A difference was not observed between the PLGA group and control group. Additionally, the PLGA-FTY720 group achieved a higher score than the PLGA group. Thus, treatment with PLGA-FTY720/NSCs resulted in the best functional recovery, and FTY720 exerted a positive effect on SCI recovery.

\subsection{Histological observations}

After SCI, cystic cavities of varying sizes appear in the injured area, leading to adverse effects on spinal cord regeneration. We performed haematoxylin and eosin staining on longitudinal sections after SCI to evaluate the effects of the different treatments on reducing cavity formation. As shown in Fig. 7, cystic cavities of different sizes were observed in all four groups at 6 weeks post-injury. A significant difference in the average cavity area was not observed between the PLGA and PLGA/FTY720 groups; however, compared with the control group, both groups had smaller cavity areas. Furthermore, the PLGAFTY720/NSC group had the smallest average cavity area among all four groups. Functionally mature neurons play an important role in SCI recovery. An anti-NeuN antibody was chosen to evaluate the mean number of positive neurons per field using immunofluorescence staining. As shown in Fig. 8, few NeuN-positive cells were detected in the control group. The PLGA and PLGA/FTY720 groups exhibited more NeuN-positive cells than the control group, and the PLGA/FTY720 group showed more positive cells than the PLGA group. The greatest number of NeuN-positive cells was observed in the PLGAFTY720/NSC group, and the difference was statistically significant.

\section{Discussion}

The restoration of neural function after SCI is always a key problem in the treatment of SCI. ${ }^{3,4}$ The discontinuous ends produced by damage substantially reduce the speed and effect of restoration. ${ }^{34,35}$ Biomaterial scaffolds provide a structural basis for the growth and migration of nerve cells in injuryinduced lesions, exerting a positive effect on the recovery and restoration of nerve function after injury. ${ }^{36-38}$ However, because the local inflammatory microenvironment and other adverse factors after SCI inhibit the differentiation of NSCs into neurons, the application of biological materials alone is unable to achieve a better therapeutic effect. ${ }^{3940}$ In addition, the number of endogenous NSCs is too limited to differentiate into a sufficient number of neurons. Therefore, the application of biomaterials loaded with drugs and NSCs to treat SCI has good 

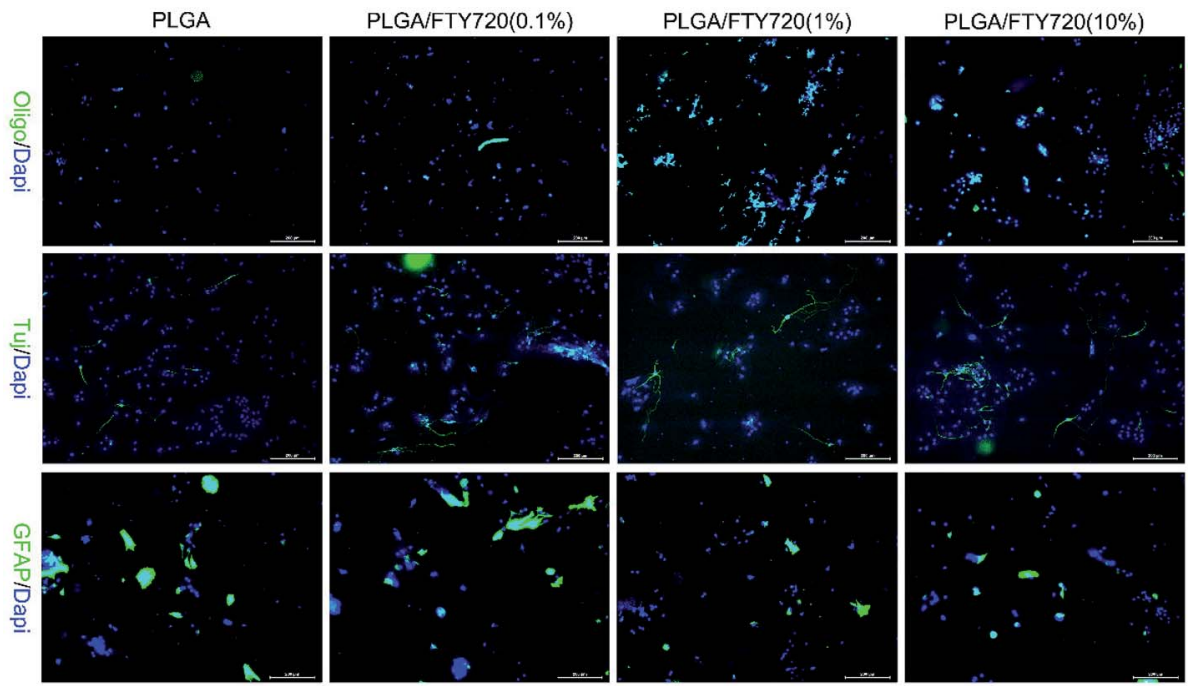

Fig. 4 Differentiation of NSCs. Immunofluorescence staining of differentiated neurons, astrocytes and oligodendrocytes in different groups. Immunostaining was conducted using antibodies against Oligo (green) to label oligodendrocytes, Tuj-1 (green) to label neurons, and GFAP (green) to label astrocytes, as well as DAPI (blue) to label nuclei. The scale bar represents $200 \mu \mathrm{m}$.

research prospects and therapeutic effects. FTY720 is an FDAapproved immunosuppressant for multiple sclerosis with a clear protective effect on the central nervous system. ${ }^{12}$ However, few studies have examined the effect of this drug on SCI, and most FTY720 delivery methods involve oral administration. ${ }^{41}$ The oral administration of FTY720 exerts some adverse effects, as mentioned in previous studies..$^{16,17}$

In our study, we synthesized electrospun microfibres to carry drugs and cells. Electron microscopy showed that the PLGA/ FTY720 fibres had a smaller diameter than PLGA fibres, and the pore structures in fibres provide space for nerve cells to proliferate and differentiate inside the scaffolds. ${ }^{22,42}$ In addition, PLGA/FTY720 microfibres had a smaller water contact angle, which indicates better hydrophily. ${ }^{43}$ Based on our results, PLGA modified by FTY720 substantially improved the hydrophilicity of the material, which compensates for the challenges associated with the hydrophobicity of PLGA materials. ${ }^{44} \mathrm{~A}$ potential explanation for these phenomena is that the PLGA solution mixed with FTY720 has better ductility and consistency. ${ }^{43}$ We speculate that these characteristics enhance cell adhesion and growth.

Next, we explored the effects of fibres on the proliferation and differentiation of NSCs and the appropriate mass ratio for local treatment by culturing NSCs with different mass ratios of
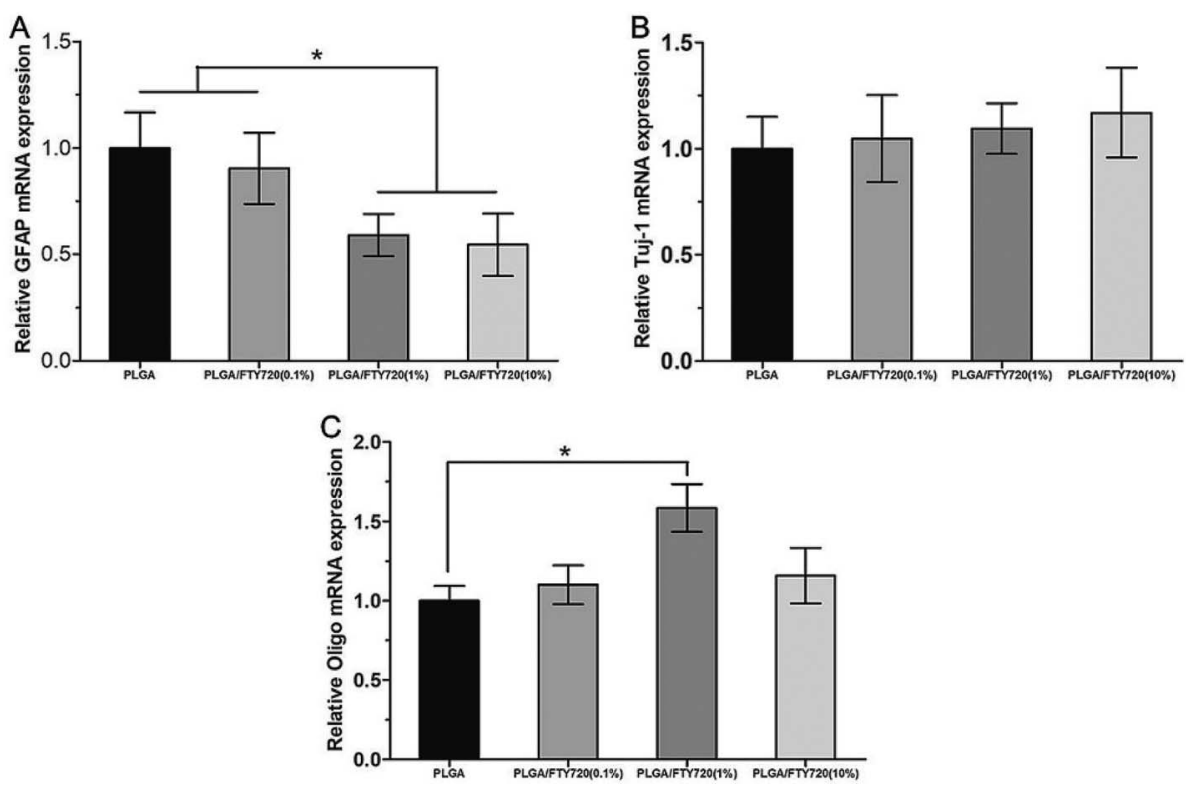

Fig. 5 qPCR analysis of GFAP (A), Tuj-1 (B) and Oligo (C) expression after NSCs were cultured for 7 days with the different scaffolds. $(* P<0.05, n$ $=3)$. 


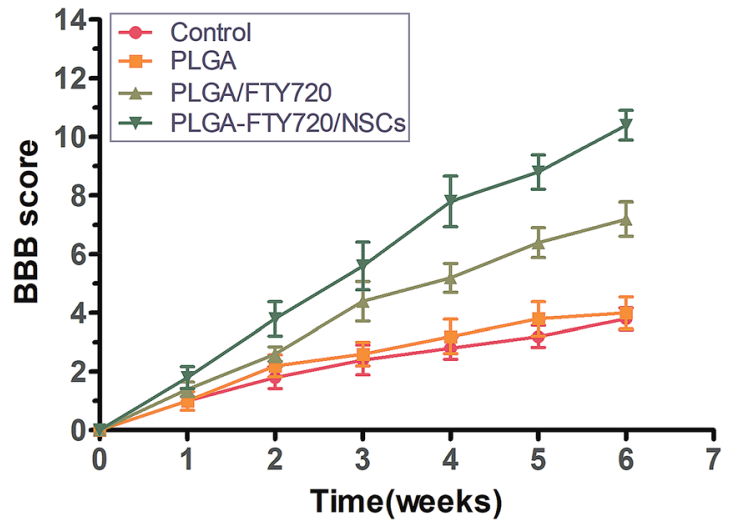

Fig. 6 BBB scores of $\mathrm{SCl}$ model rats. $(* P<0.05, n=5)$.
FTY720 in vitro. Increased proliferation of NSCs was observed with a higher PLGA/FTY720 mass ratio in a certain range. Thus, PLGA/FTY720 significantly increased the growth of NSCs at the appropriate mass ratio. However, PLGA/FTY720 microfibres at much higher mass ratio (10\%) significantly inhibited the proliferation of NSCs. Additionally, the inhibitory effect of a high ratio of FTY720 on NSC proliferation may be based on the cytotoxicity of the drug itself. ${ }^{\mathbf{4 1}}$ The results of cell differentiation assays showed that PLGA/FTY720 significantly inhibited the differentiation of NSCs into astrocytes and promoted the formation of neurons and oligodendrocytes. In the process of functional reconstruction after SCI, neurons subsequently differentiate into functional neurons and play a role in signal transmission, whereas oligodendrocytes promote the regeneration of the myelin sheath and axons, which exert a positive
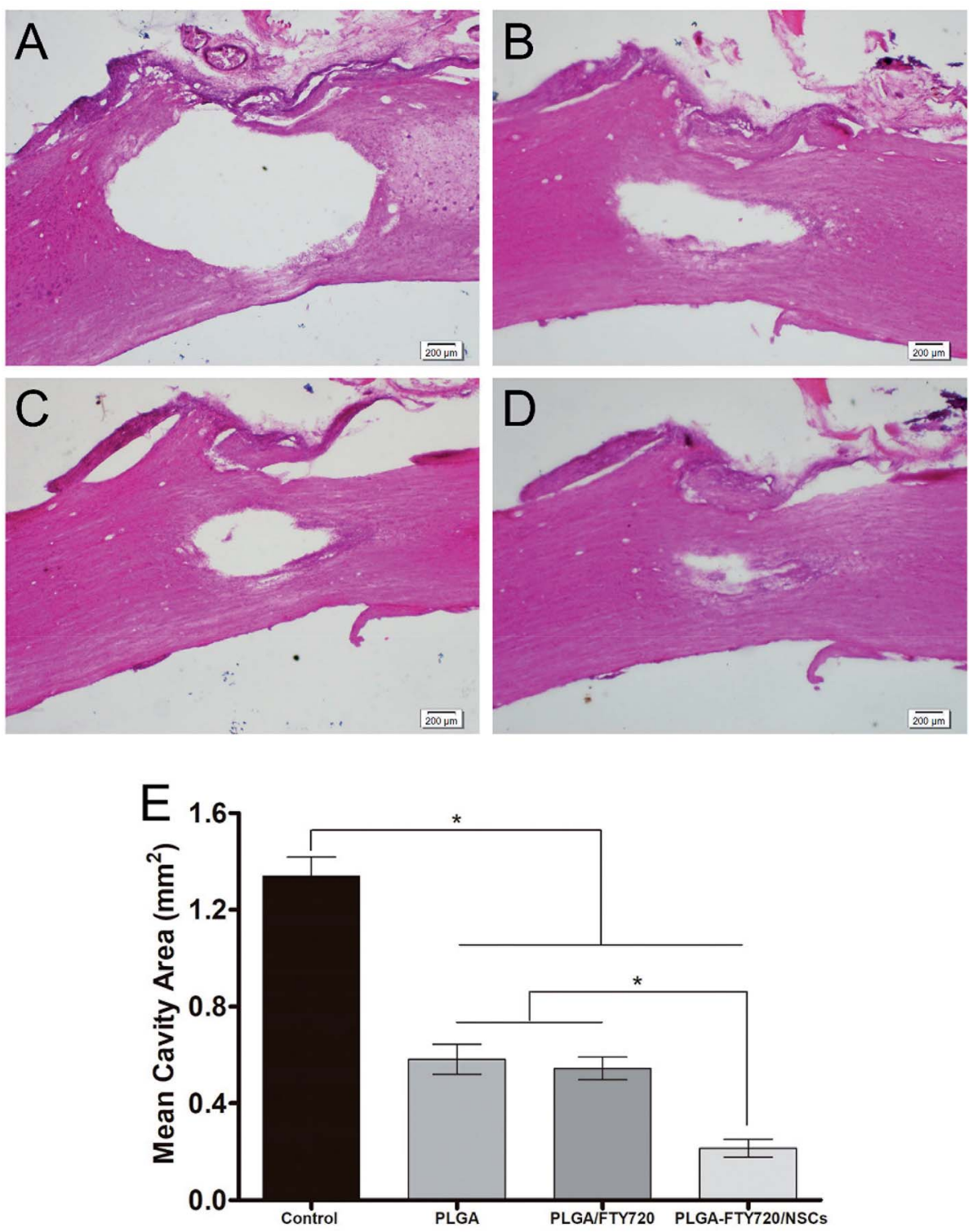

Fig. 7 HE staining of spinal cord tissues at 6 weeks after the operation. Control group (A), PLGA group (B), PLGA/FTY720 group (C) and PLGAFTY720/NSC group (D). The scale bar represents $200 \mu \mathrm{m}$. (E) The average cavity areas in spinal cord tissues from different groups. $(* P<0.05, n=$ 3). 

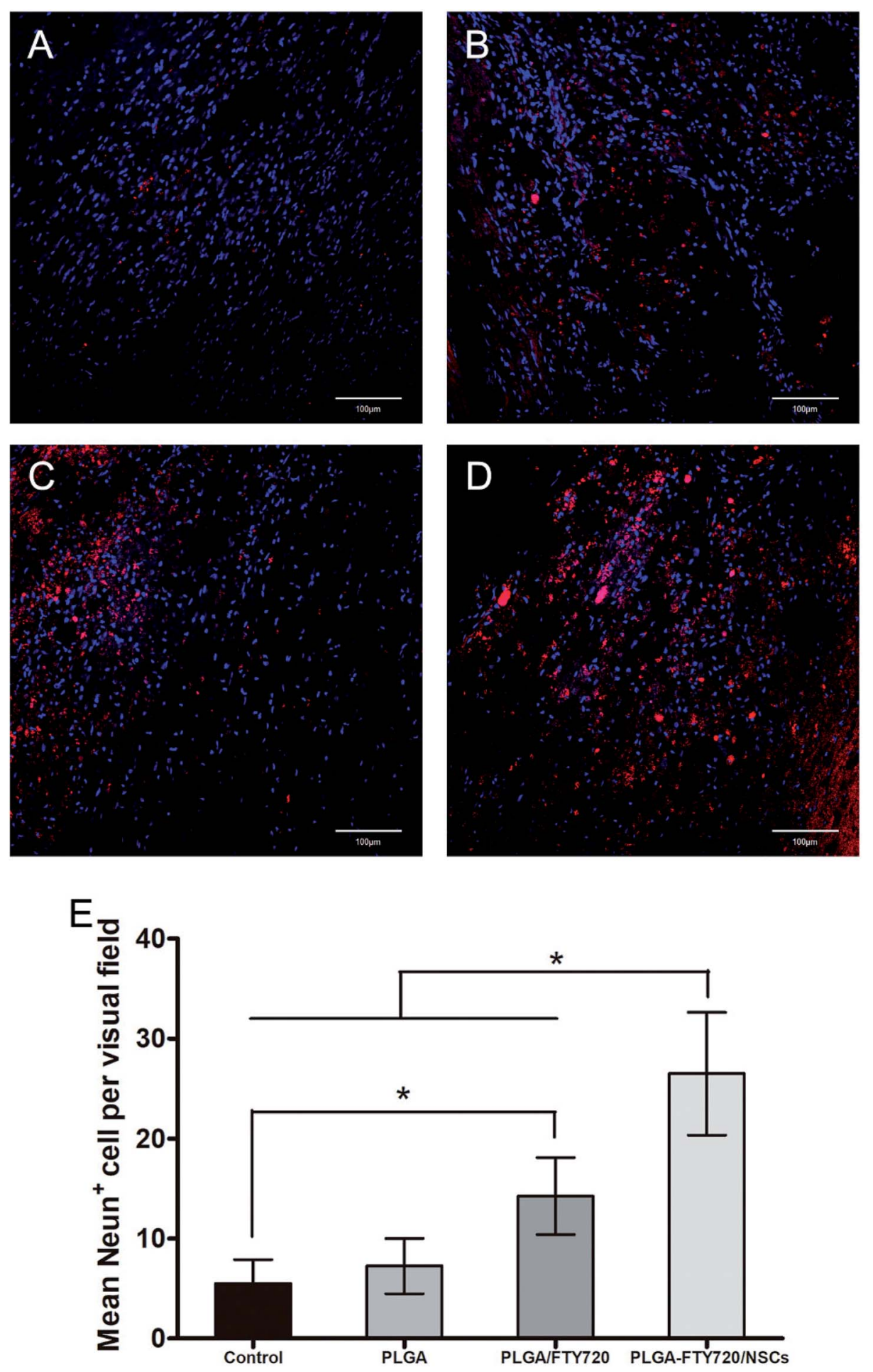

Fig. 8 Immunofluorescence staining of neurons in the injury site after SCl. Images of spinal cord tissues from the control group (A), PLGA group (B), PLGA/FTY720 group (C) and PLGA-FTY720/NSC group (D) are shown. (E) Mean number of NeuN-positive cells per visual field.

effect on functional recovery. ${ }^{45,46}$ The results of cell differentiation assays were consistent with the expected results for spinal cord recovery, suggesting that PLGA/FTY720 exerted a positive effect on functional reconstruction. Although qPCR and immunofluorescence staining did not reveal obvious effects of PLGA/FTY720 on NSC differentiation into neurons, the proper mass ratio of PLGA/FTY720 nanofibres induced NSC differentiation into oligodendrocytes, which promote axon regeneration. ${ }^{29,47}$ Moreover, FTY720 significantly inhibited the differentiation of astrocytes, which would lead to glial scar formation. ${ }^{48}$ PLGA/FTY720 nanofibres with a mass ratio of $1 \%$ noticeably increased oligodendrocyte differentiation and 
inhibited astrocyte differentiation. Therefore, we selected PLGA/FTY720 nanofibres with a mass ratio of $1 \%$ as the intervention mass ratio in the subsequent experiments.

We established a T9 spinal cord transection rat model in our study to better assess the effect of treatment with PLGA-FTY720/ NSC microfibre scaffolds in vivo. The electrospun microfibres were processed into cylinders, which are able to be properly placed in the injured area. Different scaffolds were implanted into the lesion site. Based on the BBB scores, PLGA-FTY720/ NSCs promoted a distinct improvement in hind limb motor function recovery. The scaffolds composed of PLGA/FTY720 and PLGA alone also restored hind limb motor function, but not as effectively as scaffolds prepared using PLGA/FTY720 loaded with NSCs. The difference between the groups with and without FTY720 and NSCs indicated that FTY720 exerted beneficial effects on SCI recovery and that NSCs are very important for SCI recovery.

HE staining showed much smaller mean cavity areas of the PLGA/FTY720 and PLGA groups than the control group, indicating that the scaffolds and FTY720 decreased the cavity area. In addition, the cavity was smaller in animals implanted with PLGA/FTY720 scaffolds than with PLGA scaffolds. The PLGA group had a smaller cavity area than the control group, possibly because the PLGA scaffolds provided structural support for cell growth. ${ }^{38}$ The PLGA/FTY720 group had a smaller cavity area than the PLGA group, which may be related to the effect of FTY720 on promoting the proliferation and differentiation of NSCs. Our previous experiment showed that PLGA/FTY720 distinctly promotes NSC proliferation and differentiation into functional cells in vitro, consistent with the results of HE staining. The minimum cavity area of the PLGA-FTY720/NSCs group also suggested that FTY720 with NSCs exerted a much greater positive effect on reducing the cavity area after SCI. More NSCs differentiated into neurons and more cells proliferated and differentiated, which further reduced the cavity area. Neurons in the lesion area can further differentiate into functional neurons and play a role in signal transmission, which is very important for functional recovery. The number of neurons is important when evaluating the recovery after SCI, as it serves as an indicator of the treatment effect. ${ }^{25}$ Immunofluorescence staining showed that the intervention with PLGA-FTY720/NSCs significantly increased the formation of new neurons and the PLGA/ FTY720 group contained more neurons in the injured area than the PLGA group. Based on the immunofluorescence staining, NSCs played an important role in neuron formation and FTY720 promoted NSC differentiation. Our results confirmed that electrospun PLGA nanofibre scaffolds loaded with FTY720 and NSCs exert a positive effect on SCI and promote the recovery process.

Compared with other studies using drug-loaded polymer scaffolds for SCI repair and regeneration, we selected FTY720 as the intervention drug. The therapeutic effect was similar to the experimental results obtained using methylprednisolone, growth factors and other interventions, which exerted a positive effect on promoting the recovery of nerve function. ${ }^{49,50}$ PLGA was chosen as the scaffold material in our study. Some studies have used materials with better biological characteristics, such as PEG, graphene and other materials. ${ }^{21,50}$ However, PLGA still has the advantages of a mature preparation technology and lower cost. Although our study has similarities to other studies, the effect of PLGA/FTY720 scaffolds with different mass ratios on the proliferation and differentiation of NSCs had not been explored. In our study, we cultured NSCs on PLGA/FTY720 scaffolds with different mass ratios to explore the effects of different drug doses on the proliferation and differentiation of NSCs. FTY720/PLGA scaffolds exerted a significant cytotoxic effect on NSCs when the mass ratio reached $10 \%$, indicating that FTY 720 / PLGA scaffolds only promoted proliferation and differentiation within a certain range of mass ratios, but exerted a cytotoxic effect on NSCs when the mass ratio exceeded a certain value. Thus, the mass ratio must be strictly controlled in the application of PLGA/FTY720 stents to treat SCI. Therefore, the effects of PLGA/FTY720 scaffolds with different mass ratios should be examined using cell-based experiments. According to the results of the cell-based experiment, PLGA/FTY720 scaffolds induced the highest levels of NSC proliferation and differentiation at a mass ratio of $1 \%$; therefore, we selected $1 \%$ PLGA/FTY720 scaffolds for the subsequent experiments. Additionally, few studies have used NSCs together with PLGA/FTY720 scaffolds. In our study, we loaded NSCs on PLGA/FTY720 scaffolds and investigated the effects of PLGA/FTY720 scaffolds combined with NSCs on the recovery of SCI by constructing a total resection model in rats. PLGA/FTY720 scaffolds combined with NSCs exerted better therapeutic effect than the stents alone, indicating the importance and value of NSCs in the treatment of SCI.

\section{Conclusions}

In summary, we prepared electrospun PLGA scaffolds to carry FTY720 and NSCs. The proper mass ratio of PLGA/FTY720 exerts significant positive effects on NSC proliferation and differentiation in vitro, suggesting that the local application of PLGA/ FTY720 scaffolds is an effective treatment for SCI recovery. Based on the results from the animal experiment, the scaffold effectively promotes the recovery of nerve function after implantation into the rat spinal cord transection model. Therefore, FTY720 was properly mixed with PLGA. Moreover, the immobilization of NSCs on electrospun PLGA/FTY720 scaffolds may have potential applications as a nerve implant for treating SCI.

\section{Conflicts of interest}

The authors have no conflicts of interest to declare.

\section{Acknowledgements}

This study was supported by grants from the National Natural Science Foundation of China, No. 31572217, and the Jilin 
Provincial Educational 135 Science and Technology Project, JJKH20180224KJ and JJKH20180209KJ.

\section{References}

1 D. C. Baptiste and M. G. Fehlings, Prog. Brain Res., 2007, 161, 217-233.

2 I. C. Maier and M. E. Schwab, Philos. Trans. R. Soc., B, 2006, 361, 1611-1634.

3 C. Fan, X. Li, Y. Zhao, Z. Xiao, W. Xue, J. Sun, X. Li, Y. Zhuang, Y. Chen and J. Dai, Biomater. Sci., 2018, 6, 1723-1734.

4 H. Sabelstrom, M. Stenudd and J. Frisen, Exp. Neurol., 2014, 260, 44-49.

5 L. Harris, O. Zalucki, M. Piper and J. I. Heng, Stem Cells Int., 2016, 2016, 9745315.

6 R. Covacu and L. Brundin, Neuroscientist, 2017, 23, 27-39.

7 A. M. Stessin, D. B. Gursel, A. Schwartz, B. Parashar, F. G. Kulidzhanov, A. M. Sabbas, J. Boockvar, D. Nori and A. G. Wernicke, Neurosci. Lett., 2012, 516, 253-258.

8 A. Mantovani, M. A. Cassatella, C. Costantini and S. Jaillon, Nat. Rev. Immunol., 2011, 11, 519-531.

9 M. Pelletier, A. Micheletti and M. A. Cassatella, J. Leukocyte Biol., 2010, 88, 1163-1170.

10 J. P. Magnusson, C. Goritz, J. Tatarishvili, D. O. Dias, E. M. Smith, O. Lindvall, Z. Kokaia and J. Frisen, Science, 2014, 346, 237-241.

11 V. E. Miron, A. Schubart and J. P. Antel, J. Neurol. Sci., 2008, 274, 13-17.

12 A. Kimura, T. Ohmori, R. Ohkawa, S. Madoiwa, J. Mimuro, T. Murakami, E. Kobayashi, Y. Hoshino, Y. Yatomi and Y. Sakata, Stem Cells, 2007, 25, 115-124.

13 Y. Sun, F. Hong, L. Zhang and L. Feng, Br. J. Pharmacol., 2016, 173, 2793-2807.

14 Y. Zhao, D. Shi, K. Cao, F. Wu, X. Zhu, S. Wen, Q. You, K. Zhang, L. Liu and H. Zhou, J. Leukocyte Biol., 2018, 103, 107-118.

15 W. B. Rolland 2nd, A. Manaenko, T. Lekic, Y. Hasegawa, R. Ostrowski, J. Tang and J. H. Zhang, Acta Neurochir., 2011, 111(suppl.), 213-217.

16 J. Zhang, A. Zhang, Y. Sun, X. Cao and N. Zhang, Tohoku J. Exp. Med., 2009, 219, 295-302.

17 N. Manouchehri, O. Mirmosayyeb, S. Badihian and V. Shaygannejad, Mult. Scler. Relat. Disord., 2018, 19, 121123.

18 C. Chen, M. L. Zhao, R. K. Zhang, G. Lu, C. Y. Zhao, F. Fu, H. T. Sun, S. Zhang, Y. Tu and X. H. Li, J. Biomed. Mater. Res., Part A, 2017, 105, 1324-1332.

19 W. Zhao, J. Li, K. Jin, W. Liu, X. Qiu and C. Li, Mater. Sci. Eng., C, 2016, 59, 1181-1194.

20 C. Gumera, B. Rauck and Y. Wang, J. Mater. Chem., 2011, 21, 7033-7051.

21 D. R. Bienek, K. M. Hoffman and W. Tutak, J. Mater. Sci.: Mater. Med., 2016, 27, 146.

22 L. E. Sperling, K. P. Reis, L. G. Pozzobon, C. S. Girardi and P. Pranke, J. Biomed. Mater. Res., Part A, 2017, 105, 13331345.
23 A. Raspa, A. Marchini, R. Pugliese, M. Mauri, M. Maleki, R. Vasita and F. Gelain, Nanoscale, 2016, 8, 253-265.

24 J. Xue, J. Xie, W. Liu and Y. Xia, Acc. Chem. Res., 2017, 50, 1976-1987.

25 C. Liu, Y. Huang, M. Pang, Y. Yang, S. Li, L. Liu, T. Shu, W. Zhou, X. Wang, L. Rong and B. Liu, PLoS One, 2015, 10, e0117709.

26 N. J. Schaub, C. D. Johnson, B. Cooper and R. J. Gilbert, J. Neurotrauma, 2016, 33, 1405-1415.

27 B. Vigani, S. Rossi, G. Sandri, M. C. Bonferoni and F. Ferrari, Neural Regener. Res., 2017, 12, 1786-1790.

28 M. R. Casanova, R. L. Reis, A. Martins and N. M. Neves, Adv. Exp. Med. Biol., 2018, 1058, 247-263.

29 B. Tan, Z. Luo, Y. Yue, Y. Liu, L. Pan, L. Yu and Y. Yin, Stem Cells Int., 2016, 2016, 9671732.

30 A. Das, D. A. Barker, T. Wang, C. M. Lau, Y. Lin and E. A. Botchwey, PLoS One, 2014, 9, e101276.

31 T. Wang, J. Krieger, C. Huang, A. Das, M. P. Francis, R. Ogle and E. Botchwey, Drug Delivery Transl. Res., 2015, 6, 96-104.

32 D. T. Bowers, C. E. Olingy, P. Chhabra, L. Langman, P. H. Merrill, R. S. Linhart, M. L. Tanes, D. Lin, K. L. Brayman and E. A. Botchwey, J. Biomed. Mater. Res., Part B, 2018, 106, 555-568.

33 J. Wang, J. Wang, P. Lu, Y. Cai, Y. Wang, L. Hong, H. Ren, B. C. Heng, H. Liu, J. Zhou and H. Ouyang, Biomaterials, 2015, 62, 76-87.

34 E. D. Gomes, S. S. Mendes, H. Leite-Almeida, J. M. Gimble, R. Y. Tam, M. S. Shoichet, N. Sousa, N. A. Silva and A. J. Salgado, Biomaterials, 2016, 105, 38-51.

35 C. Wang, C. Sun, Z. Hu, X. Huo, Y. Yang, X. Liu, B. O. A. Botchway, H. Davies and M. Fang, Neurosignals, 2017, 25, 1-14.

36 D. Liang, B. S. Hsiao and B. Chu, Adv. Drug Delivery Rev., 2007, 59, 1392-1412.

37 D. R. Nisbet, J. S. Forsythe, W. Shen, D. I. Finkelstein and M. K. Horne, J. Biomater. Appl., 2009, 24, 7-29.

38 P. Sensharma, G. Madhumathi, R. D. Jayant and A. K. Jaiswal, Mater. Sci. Eng., C, 2017, 77, 1302-1315.

39 X. Li, C. Fan, Z. Xiao, Y. Zhao, H. Zhang, J. Sun, Y. Zhuang, X. Wu, J. Shi, Y. Chen and J. Dai, Biomaterials, 2018, 183, 114-127.

40 X. Li, Y. Zhao, S. Cheng, S. Han, M. Shu, B. Chen, X. Chen, F. Tang, N. Wang, Y. Tu, B. Wang, Z. Xiao, S. Zhang and J. Dai, Biomaterials, 2017, 137, 73-86.

41 Y. Zhang, X. Li, B. Ciric, C. G. Ma, B. Gran, A. Rostami and G. X. Zhang, Mol. Ther., 2017, 25, 401-415.

42 T. Fuhrmann, P. N. Anandakumaran and M. S. Shoichet, Adv. Healthcare Mater., 2017, 6(10), 1601130.

43 J. Wang and M. Windbergs, Eur. J. Pharm. Sci., 2018, 124, 7179.

44 B. L. Du, C. G. Zeng, W. Zhang, D. P. Quan, E. A. Ling and Y. S. Zeng, J. Biomed. Mater. Res., Part A, 2014, 102, 17151725.

45 D. R. Serwanski, A. L. Rasmussen, C. B. Brunquell, S. S. Perkins and A. Nishiyama, Neuroglia, 2018, 1, 91-105.

46 P. Song, X. Xia, T. Han, H. Fang, Y. Wang, F. Dong, R. Zhang, P. Ge and C. Shen, Biosci. Rep., 2018, 38(5), BSR20180303. 
47 B. Tan, J. Yu, Y. Yin, G. Jia, W. Jiang and L. Yu, Neural Regener. Res., 2014, 9, 329-336.

48 M. Pitteri, R. Magliozzi, A. Bajrami, V. Camera and M. Calabrese, Expert Opin. Pharmacother., 2018, 19, 387-395.
49 S. Bin, N. Zhou, J. Pan, F. Pan, X. F. Wu and Z. H. Zhou, Drug Dev. Ind. Pharm., 2017, 43, 1033-1041.

50 S. Pan, Z. Qi, Q. Li, Y. Ma, C. Fu, S. Zheng, W. Kong, Q. Liu and X. Yang, Artif. Cells, Nanomed., Biotechnol., 2019, 47, 651-664. 